\title{
$L(2,1)$-Labeling of the Strong Product of Paths and Cycles
}

\author{
Zehui Shao $^{1,2}$ and Aleksander Vesel ${ }^{3}$ \\ ${ }^{1}$ School of Information Science and Technology, Chengdu University, Chengdu 610106, China \\ ${ }^{2}$ Key Laboratory of Pattern Recognition and Intelligent Information Processing, \\ Institutions of Higher Education of Sichuan Province, Sichuan 610106, China \\ ${ }^{3}$ Faculty of Natural Sciences and Mathematics, University of Maribor, Koroška Cesta 160, 2000 Maribor, Slovenia
}

Correspondence should be addressed to Zehui Shao; zshao@cdu.edu.cn

Received 21 September 2013; Accepted 24 October 2013; Published 24 February 2014

Academic Editors: Y. Wang and S. Xiang

Copyright (c) 2014 Z. Shao and A. Vesel. This is an open access article distributed under the Creative Commons Attribution License, which permits unrestricted use, distribution, and reproduction in any medium, provided the original work is properly cited.

\begin{abstract}
An $L(2,1)$-labeling of a graph $G=(V, E)$ is a function $f$ from the vertex set $V(G)$ to the set of nonnegative integers such that the labels on adjacent vertices differ by at least two and the labels on vertices at distance two differ by at least one. The span of $f$ is the difference between the largest and the smallest numbers in $f(V)$. The $\lambda$-number of $G$, denoted by $\lambda(G)$, is the minimum span over all $L(2,1)$-labelings of $G$. We consider the $\lambda$-number of $P_{n} \otimes C_{m}$ and for $n \leq 11$ the $\lambda$-number of $C_{n} \otimes C_{m}$. We determine $\lambda$-numbers of graphs of interest with the exception of a finite number of graphs and we improve the bounds on the $\lambda$-number of $C_{n} \otimes C_{m}$, $m \geq 24$ and $n \geq 26$.
\end{abstract}

\section{Introduction}

The Frequency Assignment Problem (FAP) requires assigning frequencies to transmitters in a wireless network. In a broadcasting network, each transmitter is assigned a frequency channel for its transmissions. Two transmissions can interfere if their channels are too close. That means that even if two transmitters use different channels, there still may be interference if the two transmitters are located close to each other $[1,2]$.

The spectrum of frequencies gets more and more scarce because of increasing demands, both civil and military. Thus the task is to minimize the span of frequencies while avoiding interference. One of the graph-theoretical models of FAP which is well elaborated is the concept of distanceconstrained labeling of graphs [1]. Many variants of this concept have been proposed; however, the $L(2,1)$-labeling problem where adjacent vertices must be assigned colors of distance at least two apart and vertices of distance two must be assigned different colors has attracted the most of interest $[3,4]$.
An $L(2,1)$-labeling of a graph $G$ is a function $f$ from the vertex set $V(G)$ to the set of nonnegative integers $C$ (called labels or colors) such that for any two vertices $u$ and $v$

$$
\begin{array}{ll}
|f(u)-f(v)| \geq 2 & \text { if } d(u, v)=1, \\
|f(u)-f(v)| \geq 1 & \text { if } d(u, v)=2 .
\end{array}
$$

A $k$ - $L(2,1)$-labeling is a $L(2,1)$-labeling of $G$ such that $C=\{0, \ldots, k\}$. An optimal $L(2,1)$-labeling of $G$ is a $k-L(2,1)$ labeling with $k$ smallest possible. The largest label used by an optimal $L(2,1)$-labeling is called the $\lambda$-number of $G$ and denoted by $\lambda(G)$.

There is a number of studies on the algorithms for $L(2,1)$ labeling problem $[1,5,6]$. It is known to be NP-hard for general graphs [4]. Even for some relatively simple families of graphs such as planar graphs, bipartite graphs, chordal graphs [5], and graphs of treewidth two [7], the problem is also NPhard.

Product graphs are considered in order to gain global information from the factor graphs [8]. Many interesting wireless networks are based on product graphs with simple factors, such as paths and cycles. In particular, any square 


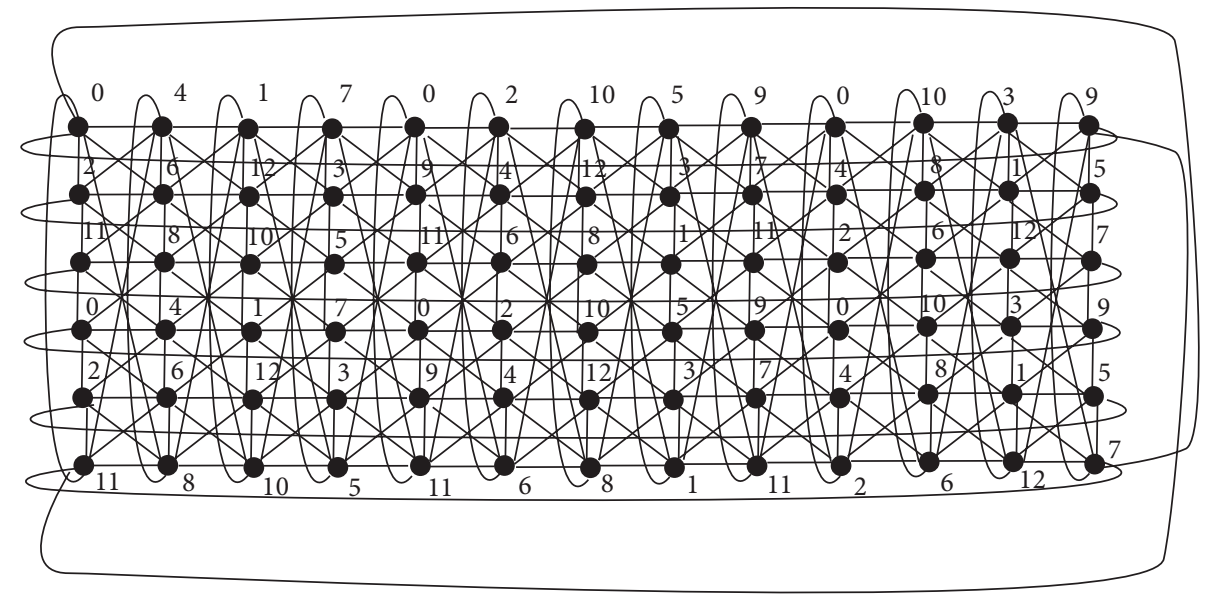

FIGURE 1: Strong product $C_{6} \otimes C_{13}$ with a $12-L(2,1)$-labeling.

grid (resp., torus) is the Cartesian product of two paths (resp., cycles) and any octagonal grid (resp., torus) is the strong product of two paths (resp., cycles) [9]. For the Cartesian product of these factors the $\lambda$ numbers have been completely determined [10-12], while for the strong and the direct product only partial results have been found [13-15].

The paper is organized as follows. In Section 2, we give definitions and concepts needed in this paper. We also report on the known results for the $\lambda$ numbers of the graphs of interest. In Section 3, two main computer search methods applied in the paper are described: the dynamic algorithm and the SAT reduction. Finally, in Section 4, we present the results on the $\lambda$-number of $P_{n} \otimes C_{m}$ and the $\lambda$-number of $C_{n} \otimes C_{m}$.

\section{Preliminaries and Previous Results}

For a graph $G=(V, E), V(G)$ and $E(G)$ are the sets of vertices and edges of $G$, respectively. A directed graph $D$ consists of vertices $V(D)$ together with a set of $\operatorname{arcs} A(D) \subseteq V(D) \times V(D)$. We write $G$ also to stand for the vertex set of the graph $G$. In this paper, only directed and undirected graphs without multiple edges or loops are considered.

The strong product of graphs $G$ and $H$ is the graph $G \otimes$ $H$ with vertex set $G \times H$ and $\left(x_{1}, x_{2}\right)\left(y_{1}, y_{2}\right) \in E(G \otimes H)$ whenever $x_{1} y_{1} \in E(G)$ and $x_{2}=y_{2}$, or $x_{2} y_{2} \in E(H)$ and $x_{1}=$ $y_{1}$, or $x_{1} y_{1} \in E(G)$ and $x_{2} y_{2} \in E(H)$. The strong product is commutative and associative, having the trivial graph as a unit (cf. [8]). The subgraph of $G \otimes H$ induced by $u \times V(H)$ is isomorphic to $H$. It is called an $H$-fiber and denoted by $H^{u}$.

The path $P_{n}$ is the graph whose vertices are $0,1, \ldots$, $n-1$ and for which two vertices are adjacent precisely if their difference is \pm 1 . For an integer $n \geq 3$, the cycle of length $n$ is the graph $C_{n}$ whose vertices are $0,1, \ldots, n-1$ and whose edges are the pairs $i, i+1$, where the arithmetic is done modulo $n$. Note that the strong product $C_{6} \otimes C_{13}$, depicted in Figure 1, can be regarded as a graph composed of six copies of $C_{13}$ (denoted by $C_{13}^{0}, \ldots, C_{13}^{5}$ ) or a graph composed of 13 copies of $C_{6}$ (denoted by $C_{6}^{0}, \ldots, C_{6}^{12}$ ).
A walk in a directed graph $D$ is a sequence of (not necessarily distinct) vertices $v_{1}, v_{2}, \ldots, v_{n}$ such that $v_{i} v_{i+1} \in$ $A(D)$ for $1,2, \ldots, n-1$. If $v_{1}=v_{n}$, we say it is a closed walk.

If $P$ is a path (resp., walk), then its length is its number of edges (resp., arcs).

The following simple lemma is well known.

Lemma 1. If $H$ is a subgraph of $G$, then $\lambda(H) \leq \lambda(G)$.

Let $f$ denote a $k$ - $L(2,1)$-labeling of $C_{n} \otimes G$. We denote by $f_{i, p}$ the restriction of $f$ to $G^{i}, G^{i+1}, \ldots, G^{i+p-1}, i=0,1, \ldots, n-1$ and $p=1, \ldots, n-p$. Note that $G^{i}$ is isomorphic to $G$; that is, $G^{i}$ is the subgraph of $G \otimes C_{n}$ induced by $V(G) \times i$. We will also write $f_{i}$ for $f_{i, 1}$.

The following lemma provides an upper bound for the $\lambda$ number of the strong product of a graph with a cycle.

Lemma 2. Let $t \geq 1$ and $f$ be a $k-L(2,1)$-labeling of $C_{n} \otimes G$. If $f_{0, p}$ is a $k-L(2,1)$-labeling of $C_{p} \otimes G$, then $\lambda\left(C_{n+(t-1) p} \otimes G\right) \leq k$.

Proof. Let $f^{\prime}$ be a function from $V\left(C_{n+(t-1) p} \otimes G\right)$ onto the set $\{0,1, \ldots, k\}$ and $f_{i}^{\prime}$ the restriction of $f^{\prime}$ to $V\left(G^{i}\right)$. The function $f^{\prime}$ is defined as follows:

$$
f_{i}^{\prime}= \begin{cases}f_{i}, & i<n \\ f_{(i-n) \bmod p} & i \geq n .\end{cases}
$$

It is not difficult to see that $f^{\prime}$ is a $k$ - $L(2,1)$-labeling of $C_{n+(t-1) p} \otimes G$.

Given two integers $r$ and $s$, let $S(r, s)$ denote the set of all nonnegative integer combinations of $r$ and $s$ :

$$
S(r, s)=\left\{\alpha r+\beta s: \alpha, \beta \in Z^{+}\right\} .
$$

We will need the result of Sylvester [16].

Lemma 3. If $r, s>1$ are relatively prime integers, then $t \in$ $S(r, s)$ for all $t \geq(s-1)(r-1)$. 


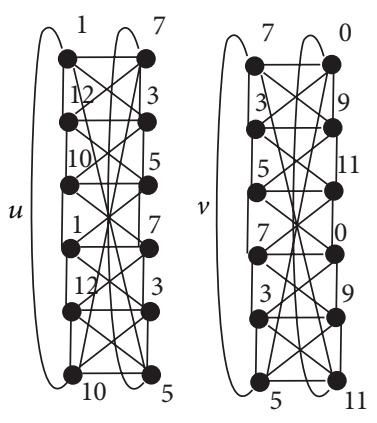

(a)

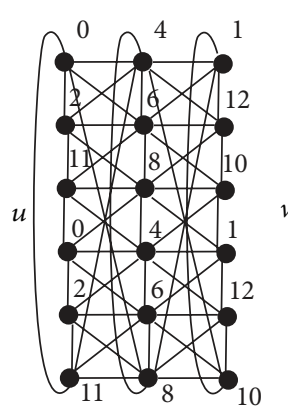

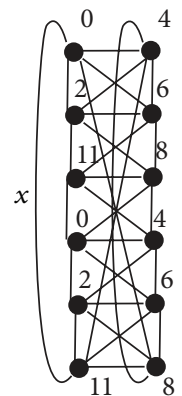

(b)

Figure 2: Two vertices of $D_{6,12}$ (a) and two vertices of $V_{6,12}^{3}$ with a vertex of $D_{6,12}^{2}$ (b).

Some partial results on the $\lambda$-number for the strong products of two cycles are given in [15].

Theorem 4. Let $m \geq 3$. Then

$$
\lambda\left(C_{3} \otimes C_{m}\right)= \begin{cases}16, & m=3,6 \\ 14, & m=5,7,10,11,15 \\ 13, & m=9,14,18,19,22,23,27,31,35 \\ 12, & \text { otherwise. }\end{cases}
$$

Theorem 5. Let $m \geq 3$. Then

$$
\lambda\left(C_{4} \otimes C_{m}\right)= \begin{cases}19, & m=5 \\ 15, & m=4,8 \\ 14, & m=11 \\ 13, & m=7,10,14,17,20,23 \\ 11, & m \equiv 0(\bmod 6) \\ 12, & \text { otherwise. }\end{cases}
$$

Theorem 6. If $m \geq 24$ and $n \geq 36$, then $\lambda\left(C_{n} \otimes C_{m}\right) \leq 12$.

For the strong product of more than two cycles the following result presented in [14] is known.

Theorem 7. If $k \geq 1$ and $m_{0}, \ldots, m_{k-1}$ are each multiple of $3^{k}+2$, then $\lambda\left(C_{m_{0}} \otimes \cdots \otimes C_{m_{k-1}}\right)=3^{k}+1$.

\section{Computer Search}

3.1. Dynamic Algorithm. The idea is introduced in [10] in a more general framework and later used several times, for example, $[13,15]$. In order to make the paper self-contained we first describe its basic definitions and results.

We define a digraph $D_{n, k}$ as follows. Its vertices are the $k$ - $L(2,1)$-labelings of $C_{n} \otimes P_{2}$. Let $u=u_{1} u_{2}$ be a vertex of $D_{n, k}$. Then $u_{1}$ and $u_{2}$ represent the $k-L(2,1)$-labeling of $C_{n} \otimes P_{2}$ restricted to the first and second copies of $C_{n}$, respectively.
Let $u$ and $v$ be two vertices of $D_{n, k}$. Then $\widetilde{u v}$ denotes the labeling of $C_{n} \otimes P_{3}$ obtained by applying $u_{1}, u_{2}$, and $v_{2}$ to the consecutive copies of $C_{n}$. (Note that $\widetilde{u v}$ is not always a $k$ - $L(2,1)$-labeling of $C_{n} \otimes P_{3}$.) We make an arc from $u$ to $v$ in $D_{n, k}$ if and only if the following two conditions are fulfilled:

(i) $u_{2}$ equals $v_{1} ;(i i) \widetilde{u v}$ is a $k-L(2,1)$-labeling of $C_{n} \otimes P_{3}$.

Analogously, we define a digraph $D_{n, k}^{\prime}$ with the vertex set composed by $k-L(2,1)$-labelings of $P_{n} \otimes P_{2}$. In other words, if $u=u_{1} u_{2}$ is a vertex of $D_{n, k}^{\prime}$, then $u_{1}$ and $u_{2}$ represent the $k$ - $L(2,1)$-labeling of $P_{n} \otimes P_{2}$ restricted to the first and second copies of $P_{n}$, respectively. The set of $\operatorname{arcs}$ of $D_{n, k}^{\prime}$ is formed analogously as the set of arcs of $D_{n, k}$.

Figure 2(a) shows two vertices of $D_{6,12}$ denoted by $u$ and $v$. We can see that the labeling of the second copy of $C_{6}$ in $u$ equals the labeling of the first copy of $C_{6}$ in $v$. Moreover, the labeling of $u$ and the labeling of the second copy of $C_{6}$ in $v$ induce a 12- $L(2,1)$-labeling of $C_{6} \otimes P_{3}$. It follows that $A\left(D_{6,12}\right)$ admits an $\operatorname{arc}$ from $u$ to $v$.

The next theorem follows from the results presented in [10].

Theorem 8. $C_{i} \otimes C_{\ell}$ (resp., $\left.P_{i} \otimes C_{\ell}\right)$ admits a $k$-L $(2,1)$-labeling if and only if $D_{i, k}\left(\right.$ resp. $\left.D_{i, k}^{\prime}\right)$ contains a closed directed walk of length $\ell$.

The dynamic algorithm first generates all $k-L(2,1)$ labelings of $C_{i} \otimes P_{2}$ which are the vertices of $D_{i, k}$. Since a main building block $C_{i} \otimes P_{2}$ is usually relatively small, a simple method, for example, backtracking, can be applied for this step. In the next step, the set of edges of $D_{i, k}$ has to be generated. The procedure for this step is described in [15]. The described algorithm however has the time complexity $O\left(n^{2}\right)$, where $n$ denotes the number of vertices in $D_{i, k}$. Note that $n$ can be very large even for $i$ and $k$ of a moderate size. Some examples for $i$ and $k$ of interest are $\left|D_{5,14}\right|=114984000$, $\left|D_{6,11}\right|=1925760$, and $\left|D_{8,11}\right|=1072523264$. The complexity of the algorithm does not allow a computation of $A\left(D_{i, k}\right)$ in a reasonable time for these cases. We have therefore improved this method as described in the sequel.

Let $V_{i, k}^{3}$ denote the set of all $k-L(2,1)$-labelings of $C_{i} \otimes P_{3}$. If $u$ is an element of $V_{i, k}^{3}$, then $u_{1}, u_{2}, u_{3}$ denote the restriction of $u$ to the first, second, and third copies of $C_{i}$ in $C_{i} \otimes P_{3}$; 


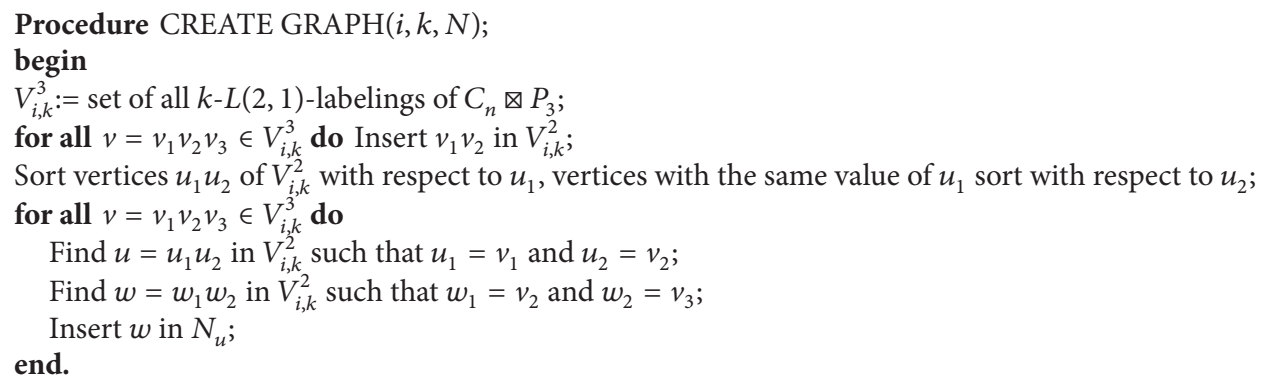

Algorithm 1

respectively. Note that $V_{i, k}^{3}$ contains symmetric labelings of $C_{i} \otimes P_{3}$, that is, if $u \in V_{i, k}^{3}$, then $v \in V_{i, k}^{3}$ exists, such that $u_{1}=v_{3}, u_{3}=v_{1}$, and $u_{2}=v_{2}$.

We now define the digraph $D_{i, k}^{2}$ as follows. Let $V\left(D_{i, k}^{2}\right)$ denote the set of $k-L(2,1)$-labelings of $C_{i} \otimes P_{2}$ obtained from $V_{i, k}^{3}$ in the following way: $x=x_{1} x_{2}$ belongs to $V\left(D_{i, k}^{2}\right)$ if and only if there exist $u, v \in V_{i, k}^{3}$ such that $u_{1}=x_{1}, u_{2}=x_{2}$, and $v_{3}=x_{1}, v_{2}=x_{2}$.

Figure 2(b) shows two vertices of $V_{6,12}^{3}$ denoted by $u$ and $v$. We can see that the labelings of the first and the second copies of $C_{6}$ in $u$ equal the third and the second copies of $C_{6}$ in $v$, respectively. It follows that $V\left(D_{6,12}^{2}\right)$ possesses the vertex $x$ comprising these two labelings.

Let $x, y \in V\left(D_{i, k}^{2}\right)$. We make an arc from $x=x_{1} x_{2}$ to $y=y_{1} y_{2}$ if and only if $x_{2}=y_{1}$ and $x_{1} x_{2} y_{2}$ belongs to $V_{i, k}^{3}$.

Note that analogous as above we can improve the method for $P_{i} \otimes C_{\ell}$. The graph obtained with this procedure (a subgraph of $D_{i, k}^{\prime}$ ) will be denoted by $D_{i, k}^{\prime 2}$ in the sequel.

For a vertex $v$ of a directed graph $D$, the number of inward (resp., outward) directed arcs from $v$ in $D$ is called an indegree (resp., outdegree) and denoted by indeg ${ }_{D(v)}$ (resp., outdeg $\left._{D(v)}\right)$.

We obtain the main result of this section.

Theorem 9. $C_{i} \otimes C_{\ell}$ (resp., $\left.P_{i} \otimes C_{\ell}\right)$ admits a $k-L(2,1)$-labeling if and only if $D_{i, k}^{2}\left(\right.$ resp., $\left.D_{i, k}^{\prime 2}\right)$ contains a closed directed walk of length $\ell$.

Proof. It is easy to see that $V\left(D_{i, k}^{2}\right) \subseteq V\left(D_{i, k}\right)$ and $A\left(D_{i, k}^{2}\right) \subseteq$ $A\left(D_{i, k}\right)$. From the definition of $V_{i, k}^{2}$ for $x \in V\left(D_{i, k}^{2}\right)$ it follows that indeg $D_{D_{i, k}}(x)>0$ and outdeg $D_{D_{i, k}}(x)>0$.

Suppose now for $y \in V\left(D_{i, k}\right)$ that indeg ${ }_{D_{i, k}}(y)>0$ and outdeg $_{D_{i, k}}(y)>0$. Since $V_{i, k}^{3}$ contains all $k$ - $L(2,1)$-labelings of $C_{i} \otimes P_{3}$ and outdeg $g_{D_{i, k}}(y)>0$, there has to be a vertex $v \in V_{i, k}^{3}$ such that $v_{1}=y_{1}$ and $v_{2}=y_{2}$. Moreover, since indeg $_{D_{i, k}}(y)>0$, there has to be a vertex $u \in V_{i, k}^{3}$ such that $u_{2}=y_{1}$ and $u_{3}=y_{2}$. It follows that $y$ belongs to $V\left(D_{i, k}^{2}\right)$. We have therefore proven that $x \in V\left(D_{i, k}^{2}\right)$ if and only if indeg $_{D_{i, k}}(x)>0$ and outdeg $D_{D_{i, k}}(x)>0$.

Analogously as above we can show that $x y$ is an $\operatorname{arc}$ in $D_{i, k}^{2}$ if and only if $x y$ is an arc in $D_{i, k}$. In other words, we can show that $D_{i, k}^{2}$ is isomorphic to subgraph of $D_{i, k}$ induced by $V_{i, k}^{2}$. It follows that $D_{i, k}^{2}$ contains a closed directed walk of length $\ell$ if and only if $D_{i, k}$ contains a closed directed walk of the same size and the proof for $D_{i, k}$ is settled.

Since the proof for $D_{i, k}^{\prime 2}$ is analogous, the proof of the theorem is complete.

The algorithm for generating the graph $D_{i, k}^{2}$ is depicted in Algorithm 1 (Procedure CREATE GRAPH).

Note that the number of vertices of $D_{i, k}^{2}$ can be much smaller than in $D_{i, k}$. Some examples for $i$ and $k$ of interest are: $\left|D_{5,14}^{2}\right|=386345700,\left|D_{6,11}^{2}\right|=12336$, and $\left|D_{8,11}^{2}\right|=8157632$. However, this reduction is not the only positive effect of the new approach. It is also of a great importance that the running time of CREATE GRAPH is $O(n \log n)$, where $n$, the number of vertices in $V_{i, k}^{3}$. In order to see this, note that the running time of an efficient sorting algorithm is also within this time bound. Moreover, this is also the running time of the duration of loop, since a single search in an ordered list with $n$ elements requires $O(\log n)$ time.

The final step of the approach is the search for closed direct walks in $D_{i, k}^{2}$. We can find these walks by applying a matrix multiplication of the adjacency matrix of $D_{i, k}^{2}$ or a breadth (depth) first search in $D_{i, k}^{2}$. Since graphs $D_{i, k}^{2}$ are relatively sparse for $i$ and $k$ of interest, the later approach has been applied in order to compute the results of this paper.

3.2. SAT Reduction for $k-L(2,1)$-Labeling. The approach is proposed in [17] for the distance-constrained labeling problem. Here we present this method adapted for $L(2,1)$-labeling.

Let $G=(V, E)$ be a graph and $k$ a positive integer. For every $v \in V$ and every $i \in\{0,1,2, \ldots, k\}$ introduce an atom $x_{v, i}$. Intuitively, this atom shows that the vertex $v$ is assigned the color $i$. Consider the following propositional formulas:

(1) for all $v \in V, \vee_{i=0}^{k} x_{v, i}$;

(2) for all $v \in V, 0 \leq i<j \leq k, \neg x_{v, i} \vee \neg x_{v, j}$;

(3) for all $v, u \in V, 0 \leq i, j \leq k$ with $d(v, u)=1$ and $|i-j|<2$ or $d(v, u)=2$ and $|i-j|<1, \neg x_{v, i} \vee \neg x_{u, j}$.

Clauses (1) and (2) ensure that each vertex is labeled with exactly one label. Clause (3) guarantees that an obtained 
TABLE 1: Values of $\lambda\left(P_{n} \otimes C_{m}\right)$.

\begin{tabular}{lllllllllll}
\hline$n \backslash m$ & 3 & 4 & 5 & 6 & 7 & 8 & 9 & 10 & 11 \\
\hline 3 & 11 & 11 & 14 & 11 & 11 & 11 & 11 & 11 & 10 \\
4 & 11 & 11 & 14 & 11 & 11 & 11 & 11 & 11 & 10 \\
5 & 12 & 11 & 14 & 11 & 11 & 11 & 12 & 12 & 10 \\
6 & 12 & 11 & 14 & 11 & 11 & 11 & 12 & 12 & 10 \\
7 & 12 & 11 & 14 & 11 & 12 & 11 & 12 & 12 & 10 \\
\hline
\end{tabular}

TABLE 2: Values of $\lambda\left(C_{n} \otimes C_{m}\right)$.

\begin{tabular}{lcccccccccccccccccccccccc}
\hline$n \backslash m$ & 5 & 6 & 7 & 8 & 9 & 10 & 11 & 12 & 13 & 14 & 15 & 16 & 17 & 18 & 19 & 20 & 21 & 22 & 23 & 24 & 25 & 26 \\
\hline 5 & $24^{\mathrm{a}}$ & $14^{\mathrm{a}}$ & 17 & 19 & 14 & 16 & 18 & 14 & 16 & 17 & 14 & 15 & 16 & 14 & 15 & 16 & 14 & 15 & 16 & 14 & 15 & 16 \\
6 & & $16^{\mathrm{a}}$ & $14^{\mathrm{a}}$ & 11 & 13 & 13 & 13 & 11 & 12 & 13 & 12 & 11 & 12 & 13 & 12 & 11 & 12 & 12 & 12 & 11 & 12 & 12 \\
7 & & & 13 & 13 & 13 & 12 & 12 & 12 & 12 & 13 & 13 & 13 & 13 & 13 & 13 & 12 & 12 & 12 & 12 & 12 & 12 & 12 \\
8 & & & 13 & 12 & 13 & 12 & 11 & 12 & 12 & 12 & 12 & 12 & 11 & 12 & 12 & 12 & 12 & 12 & 11 & 12 & 12 \\
9 & & & & 13 & $13 / 14$ & 13 & 12 & 12 & 13 & 13 & 12 & 12 & 13 & 13 & 12 & 12 & 13 & 13 & 12 & 12 & 12 \\
10 & & & & & 13 & 13 & 12 & 13 & 12 & 13 & 13 & 13 & 12 & 12 & 12 & 12 & 13 & 12 & 12 & 12 & 13 \\
11 & & & & & & 10 & 12 & 12 & 12 & 12 & 12 & 11 & 12 & 12 & 12 & 12 & 12 & 12 & 12 & 12 & 12 \\
12 & & & & & & 12 & & & & 11 & 12 & 12 & 12 & 11 & 12 & 11 & 12 & 11 & 12 & 12 & 12 & 11 & 12 & 12 \\
\hline
\end{tabular}

labeling is a $k-L(2,1)$-labeling of $G$. Therefore, the above propositional formulas transform an $L(2,1)$-labeling problem into a propositional satisfiability test (SAT). We can see that an obtained SAT instance is satisfiable if and only if $G$ admits a $k$ - $L(2,1)$-labeling.

\section{Results}

4.1. SAT Reduction. We solve the SAT instances transformed from $L(2,1)$-labeling problems described in Section 4.2 by using the software MiniSat [18]. As a result, we have obtained the $\lambda$-numbers of $P_{n} \otimes C_{m}$ presented in Table 1 and the $\lambda$ numbers of $C_{n} \otimes C_{m}$ presented in Table 2 .

The values in Table 2 marked with $a$ denote the results already obtained in [15], while the entry with $13 / 14$ means that the corresponding value is either 13 or 14 .

\section{2. $\lambda$-Labeling of $C_{n} \otimes C_{m}$}

Proposition 10. $\lambda\left(C_{5} \otimes C_{m}\right)=14$ only if $m \equiv 0(\bmod 3)$.

Proof. Note that $\lambda\left(C_{5} \otimes P_{3}\right)=14$. We can see that $\lambda\left(C_{5} \otimes P_{3}\right) \leq$ 14 from the fact that every pair of vertices $u, v \in V\left(C_{5} \otimes P_{3}\right)$ is at distance at most two. Let $f$ denote a $14-L(2,1)$-labeling of $C_{5} \otimes C_{m}$ and $f_{k}$ its restriction to $C_{5}^{k}$. Let also $L_{k}$ denote the set of labels used in $f_{k}$. Since $\lambda\left(C_{5} \otimes P_{3}\right)=14$, we have $\left|L_{k}\right|=5$ and $\left|L_{k}\right|+\left|L_{k+1}\right|=10$. Therefore, the restriction of $f$ to $C_{5}^{k+3}$ has to comprise the same set of labels as the restriction of $f$ to $C_{5}^{k}$ or, more formally, $L_{k}=L_{k+3}$. It is straightforward to see that this equality can be satisfied in $C_{5} \otimes C_{m}$ only if $m \equiv 0$ $(\bmod 3)$.
Theorem 11. Let $m \geq 5$. Then

$$
\lambda\left(C_{5} \otimes C_{m}\right)= \begin{cases}14, & m \equiv 0(\bmod 3) \\ 17, & m=7,14 \\ 19, & m=8 \\ 18, & m=11 \\ 16, & m=10,13,17,20,23,26,29 \\ 15, & \text { otherwise. }\end{cases}
$$

Proof. Note that the values for $m \leq 26$ are given in Table 2 . We can also show by using the SAT reduction that $\lambda\left(C_{5} \otimes\right.$ $\left.C_{29}\right)=16$. Since $\lambda\left(C_{5} \otimes C_{j}\right)=14$ only if $j \equiv 0(\bmod 3)$, we construct below a 14- $L(2,1)$-labeling for $C_{5} \otimes C_{3 j}, j \geq 1$, a $15-L(2,1)$-labeling for $C_{5} \otimes C_{3 j+1}, j \geq 5$, and a $15-L(2,1)$ labeling for $C_{5} \otimes C_{3 j+2}, j \geq 10$.

Let $[a, b]$ for $b \geq a$ denote the set $\{a, a+1, \ldots, b\}$.

Let $f$ denote a function from $V\left(C_{5} \otimes C_{m}\right)$ to $[0,14]$ and $f_{k}$ its restriction to $C_{5}^{k}, k \leq m-1$. Let also $L_{k}$ denote the set of labels used in $f_{k}$. If we set for $i \geq 0$ and $s \in\{0,1,2\}$ : $L_{3 i+s}:=[5 s, 5 s+4]$, then $f$ is a $14-L(2,1)$-labeling of $C_{5} \otimes C_{3 j}$ for $j \geq 1$.

Let $f^{\prime}$ denote a function from $V\left(C_{5} \otimes C_{m}\right)$ to $[0,15]$ and $f_{k}^{\prime}$ its restriction to $C_{5}^{k}, k \leq m-1$. Let also $L_{k}^{\prime}$ denote the set of labels used in $f_{k}^{\prime}$. If we set for for $0 \leq i \leq 3: L_{3 i}^{\prime}:=[15-i, 15] \cup$ $[0,3-i], L_{3 i+1}^{\prime}:=[4-i, 8-i], L_{3 i+2}^{\prime}:=[9-i, 13-i]$ and for $i \geq 4$ and $s \in\{0,1,2\}: L_{3 i+s}:=[5 s, 5 s+4]$, then $f^{\prime}$ is a $15-L(2,1)$ labeling of $C_{5} \otimes C_{3 j+1}$ for $j \geq 5$. As an example, observe the following pattern representing a $15-L(2,1)$-labeling of $C_{5} \otimes$ $\mathrm{C}_{22}$ : 


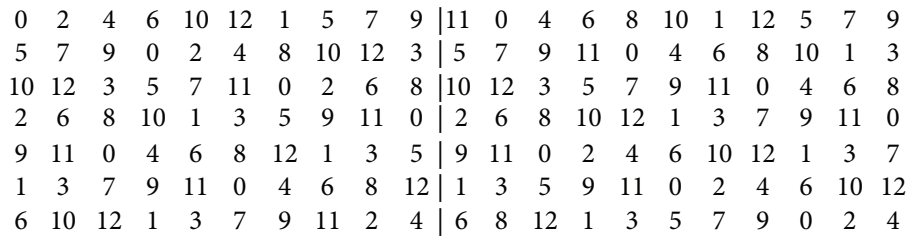

FIgURE 3: $12-L(2,1)$-labeling of $C_{7} \otimes C_{21}$.

$\begin{array}{ccccccccccccccccccccccc}15 & 4 & 9 & 14 & 3 & 8 & 13 & 2 & 7 & 12 & 1 & 6 & 11 & 0 & 5 & 10 & 0 & 5 & 10 & 0 & 5 & 10 \\ 0 & 5 & 10 & 15 & 4 & 9 & 14 & 3 & 8 & 13 & 2 & 7 & 12 & 1 & 6 & 11 & 1 & 6 & 11 & 1 & 6 & 11 \\ 1 & 6 & 11 & 0 & 5 & 10 & 15 & 4 & 9 & 14 & 3 & 8 & 13 & 2 & 7 & 12 & 2 & 7 & 12 & 2 & 7 & 12 \\ 2 & 7 & 12 & 1 & 6 & 11 & 0 & 5 & 10 & 15 & 4 & 9 & 14 & 3 & 8 & 13 & 3 & 8 & 13 & 3 & 8 & 13 \\ 3 & 8 & 13 & 2 & 7 & 12 & 1 & 6 & 11 & 0 & 5 & 10 & 15 & 4 & 9 & 14 & 4 & 9 & 14 & 4 & 9 & 14\end{array}$

Let $f^{\prime \prime}$ denote a function from $V\left(C_{5} \otimes C_{m}\right)$ to $[0,15]$ and $f_{k}^{\prime \prime}$ its restriction to $C_{5}^{k}, k \leq m-1$. Let also $L_{k}^{\prime \prime}$ denote the set of labels used in $f_{k}^{\prime \prime}$. If we set for for $0 \leq k \leq 31: L_{k}^{\prime \prime}:=L_{k}^{\prime}$ and for $i \geq 11$ and $s \in\{0,1,2\}: L_{3 i+s}^{\prime \prime}:=[5 s, 5 s+4]$, then $f^{\prime \prime}$ is a $15-L(2,1)$-labeling of $C_{5} \otimes C_{3 j+2}$ for $j \geq 10$. This assertion concludes the proof.

The following results partially depend on comprehensive constructions which provide labelings of interest. These constructions are mostly not included in this paper and can be obtained by the authors.

Proposition 12. $\lambda\left(C_{6} \otimes C_{m}\right)=11$ only if $m \equiv 0(\bmod 4)$.

Proof. The graph $D_{6,11}^{2}$ with 12336 vertices and the largest outdegree six has been computed. Since breadth first search algorithm has found only cycles of length four, Theorem 9 yields the proof.

Theorem 13. If $m \geq 6$, then

$$
\lambda\left(C_{6} \otimes C_{m}\right)= \begin{cases}11, & m \equiv 0(\bmod 4) \\ 16, & m=6 \\ 14, & m=7 \\ 13, & m=9,10,11,14 \\ 12, & \text { otherwise. }\end{cases}
$$

Proof. The results for $m=6,7,9,10,11,14$ follow from Table 2 . We can also see in Table 1 that $\lambda\left(C_{6} \otimes P_{3}\right)=11$; thus, from Lemma 1 it follows that $\lambda\left(C_{6} \otimes C_{m}\right) \geq 11$. Moreover, Proposition 12 says that $\lambda\left(C_{6} \otimes C_{m}\right)=11$ only if $m \equiv$ $0(\bmod 4)$. In order to see that $\lambda\left(C_{6} \otimes C_{m}\right) \leq 12$ for other $m$ of interest, see as an example a $12-L(2,1)$-labeling of $C_{6} \otimes C_{13}$ depicted in Figure 1.

From Lemma 2 it follows that $\lambda\left(C_{6} \otimes C_{m}\right) \leq 12$ if $m \equiv 0(\bmod 13)$. Analogously, we have found $12-L(2,1)-$ labelings of $C_{6} \otimes C_{40}, C_{6} \otimes C_{41}, C_{6} \otimes C_{29}, C_{6} \otimes C_{30}, C_{6} \otimes C_{44}$, $C_{6} \otimes C_{45}, C_{6} \otimes C_{33}, C_{6} \otimes C_{34}, C_{6} \otimes C_{48}, C_{6} \otimes C_{36}, C_{6} \otimes C_{50}$, and $C_{6} \otimes C_{38}$. Any of these labelings restricted to the first 13 copies of $C_{6}$ induces a 12- $L(2,1)$-labeling of $C_{6} \otimes C_{13}$. From Lemma 2 it follows that $\lambda\left(C_{6} \otimes C_{13 k+1}\right) \leq 12$ for $k \geq 3$, $\lambda\left(C_{6} \otimes C_{13 k+2}\right) \leq 12$ for $k \geq 3, \lambda\left(C_{6} \otimes C_{13 k+3}\right) \leq 12$ for $k \geq 2$, $\lambda\left(C_{6} \otimes C_{13 k+4}\right) \leq 12$ for $k \geq 2, \lambda\left(C_{6} \otimes C_{13 k+5}\right) \leq 12$ for $k \geq 3$, $\lambda\left(C_{6} \otimes C_{13 k+6}\right) \leq 12$ for $k \geq 3, \lambda\left(C_{6} \otimes C_{13 k+7}\right) \leq 12$ for $k \geq 2$, $\lambda\left(C_{6} \otimes C_{13 k+8}\right) \leq 12$ for $k \geq 2, \lambda\left(C_{6} \otimes C_{13 k+9}\right) \leq 12$ for $k \geq 3$, $\lambda\left(C_{6} \otimes C_{13 k+10}\right) \leq 12$ for $k \geq 2, \lambda\left(C_{6} \otimes C_{13 k+11}\right) \leq 12$ for $k \geq 3$, and $\lambda\left(C_{6} \otimes C_{13 k+12}\right) \leq 12$ for $k \geq 2$.

Since we have also found 12- $L(2,1)$-labelings of $C_{6} \otimes C_{27}$, $C_{6} \otimes C_{28}, C_{6} \otimes C_{31}, C_{6} \otimes C_{32}, C_{6} \otimes C_{35}$, and $C_{6} \otimes C_{37}$, we conclude that $\lambda\left(C_{6} \otimes C_{m}\right) \leq 12$ for $m \geq 27$ and the proof is complete.

Theorem 14. If $m \geq 7$, then

$$
\lambda\left(C_{7} \otimes C_{m}\right)=\left\{\begin{array}{cc}
13, & m=7,8,9,14,15,16,17, \\
12, & \text { otherwise. }
\end{array}\right.
$$

Proof. The results for $m \leq 26$ follow from Table 2 . We have also established the results for $m=27,28,29$ by solving the SAT instances transformed from the corresponding $L(2,1)$ labeling problems. Since $\lambda\left(C_{7} \otimes P_{7}\right)=12$ as we can see in Table 2 , it follows by Lemma 1 that $\lambda\left(C_{7} \otimes C_{m}\right) \geq 12$ for $m \geq 7$. In order to see that $\lambda\left(C_{7} \otimes C_{m}\right) \leq 12$ for other $m$ of interest, see as an example a $12-L(2,1)$-labeling of $C_{7} \otimes C_{21}$ depicted in Figure 3. This labeling restricted to the first ten copies of $C_{7}$ induces a $12-L(2,1)$-labeling of $C_{7} \otimes C_{10}$. From Lemma 2 it follows that $\lambda\left(C_{7} \otimes C_{10 k+1}\right) \leq 12, k \geq 2$, and $\lambda\left(C_{7} \otimes C_{10 k}\right) \leq 12$, $k \geq 1$.

Analogously, we have found 12-L(2,1)-labelings of $C_{7} \otimes$ $C_{22}, C_{7} \otimes C_{23}, C_{7} \otimes C_{34}, C_{7} \otimes C_{35}, C_{7} \otimes C_{36}, C_{7} \otimes C_{47}, C_{7} \otimes C_{48}$, and $C_{7} \otimes C_{49}$. Any of these labelings restricted to the first ten copies of $C_{7}$ is a 12-L(2,1)-labeling of $C_{7} \otimes C_{10}$. From Lemma 2 it follows that $\lambda\left(C_{7} \otimes C_{10 k+2}\right) \leq 12$ for $k \geq 2, \lambda\left(C_{7} \otimes C_{10 k+3}\right) \leq 12$ for $k \geq 2, \lambda\left(C_{7} \otimes C_{10 k+3}\right) \leq 12$ for $k \geq 2, \lambda\left(C_{7} \otimes C_{10 k+4}\right) \leq 12$ for $k \geq 3, \lambda\left(C_{7} \otimes C_{10 k+5}\right) \leq 12$ for $k \geq 3, \lambda\left(C_{7} \otimes C_{10 k+6}\right) \leq 12$ for $k \geq 3, \lambda\left(C_{7} \otimes C_{10 k+7}\right) \leq 12$ for $k \geq 4, \lambda\left(C_{7} \otimes C_{10 k+8}\right) \leq 12$ for $k \geq 4$, and $\lambda\left(C_{7} \otimes C_{10 k+9}\right) \leq 12$ for $k \geq 4$. 


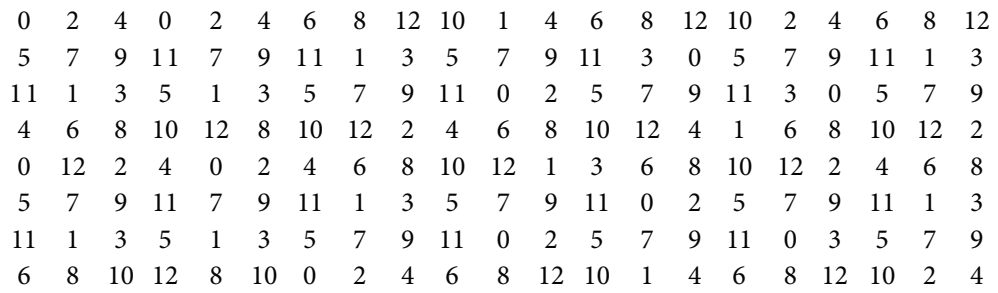

FIgURE 4: 12-L(2,1)-labeling of $C_{8} \otimes C_{21}$.

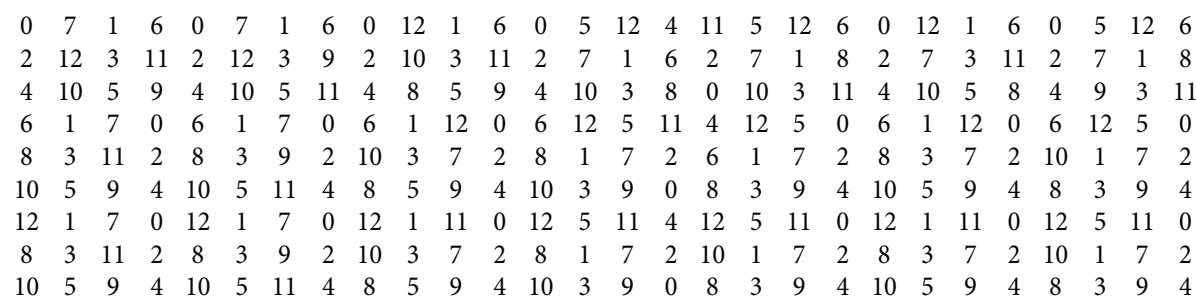

Figure 5: 12- $L(2,1)$-labeling of $C_{9} \otimes C_{28}$.

Since we have also found 12- $L(2,1)$-labelings of $C_{7} \otimes C_{27}$, $C_{7} \otimes C_{37}, C_{7} \otimes C_{38}$, and $C_{7} \otimes C_{39}$, it follows that $\lambda\left(C_{7} \otimes C_{m}\right) \leq 12$ for all graphs of interest and the proof is complete.

Proposition 15. $\lambda\left(C_{8} \otimes C_{m}\right)=11$ only if $m \equiv 0(\bmod 6)$.

Proof. The graph $D_{8,11}^{2}$ with 8157632 vertices and the largest outdegree 8 has been computed. Since breadth first search algorithm has found only cycles of length, six, twelve, and twenty-four, Theorem 9 yields the proof.

Theorem 16. If $m \geq 8$, then

$$
\lambda\left(C_{8} \otimes C_{m}\right)= \begin{cases}11, & m \equiv 0(\bmod 6) \\ 13, & m=10 \\ 12, & \text { otherwise. }\end{cases}
$$

Proof. Since $\lambda\left(C_{8} \otimes P_{7}\right)=11$ (see Table 2), it follows from Lemma 1 that $\lambda\left(C_{8} \otimes C_{m}\right) \geq 11$. Proposition 15 says that $\lambda\left(C_{8} \otimes\right.$ $\left.C_{m}\right)=11$ only if $m \equiv 0(\bmod 6)$, while the results for $m \leq$ 26 follow from Table 2 . Figure 4 shows a $12-L(2,1)$-labeling of $C_{8} \otimes C_{21}$. This labeling restricted to the first nine copies of $C_{8}$ induces a $12-L(2,1)$-labeling of $C_{8} \otimes C_{9}$. From Lemma 2 it follows that $\lambda\left(C_{8} \otimes C_{9 k+3}\right) \leq 12, k \geq 2$, and $\lambda\left(C_{8} \otimes C_{9 k}\right) \leq 12$, $k \geq 1$.

Moreover, we have found 12- $L(2,1)$-labelings of $C_{8} \otimes C_{46}$, $C_{8} \otimes C_{47}, C_{8} \otimes C_{40}, C_{8} \otimes C_{50}, C_{8} \otimes C_{24}, C_{8} \otimes C_{43}$, and $C_{8} \otimes C_{44}$. Any of these labelings restricted to the first nine copies of $C_{8}$ is a $12-L(2,1)$-labeling of $C_{8} \otimes C_{9}$. From Lemma 2 it follows that $\lambda\left(C_{8} \otimes C_{9 k+1}\right) \leq 12$ for $k \geq 5, \lambda\left(C_{8} \otimes C_{9 k+2}\right) \leq 12$ for $k \geq 5, \lambda\left(C_{8} \otimes C_{9 k+4}\right) \leq 12$ for $k \geq 4, \lambda\left(C_{8} \otimes C_{9 k+5}\right) \leq 12$ for $k \geq 5, \lambda\left(C_{8} \otimes C_{9 k+6}\right) \leq 12$ for $k \geq 2, \lambda\left(C_{8} \otimes C_{9 k+7}\right) \leq 12$ for $k \geq 4$, and $\lambda\left(C_{8} \otimes C_{9 k+8}\right) \leq 12$ for $k \geq 4$.

Since we have also found $12-L(2,1)$-labelings of $C_{8} \otimes C_{28}$, $C_{8} \otimes C_{29}, C_{8} \otimes C_{31}, C_{8} \otimes C_{32}, C_{8} \otimes C_{34}, C_{8} \otimes C_{35}, C_{8} \otimes C_{37}$,
$C_{8} \otimes C_{38}$, and $C_{8} \otimes C_{41}$, we establish the desired upper bound for all graphs of interest and the proof is complete.

Theorem 17. If $m \geq 9$, then

$$
\lambda\left(C_{9} \otimes C_{m}\right)= \begin{cases}13, & m=9,11,15,14,18,19,22,23 \\ 13 \text { or } 14, & m=10 \\ 12 \text { or } 13, & m=27,31,35 \\ 12, & \text { otherwise. }\end{cases}
$$

Proof. Since $\lambda\left(C_{9} \otimes P_{7}\right)=12$ (see Table 2), it follows from Lemma 1 that $\lambda\left(C_{9} \otimes C_{m}\right) \geq 12$. The results for $m \leq 26$ follow from Table 2 . We have also established by solving the SAT instances transformed from the corresponding $L(2,1)$ labeling problems that $\lambda\left(C_{9} \otimes C_{10}\right)$ is either 13 or 14 , while for $m=27,31,35$ the value of $\lambda\left(C_{9} \otimes C_{m}\right)$ is either 12 or 13 . Figure 5 shows a $12-L(2,1)$-labeling of $C_{9} \otimes C_{28}$. This labeling restricted to first 12 copies of $C_{9}$ induces a $12-L(2,1)$-labeling of $C_{9} \otimes C_{12}$. From Lemma 2 it follows that $\lambda\left(C_{9} \otimes C_{12 k+4}\right) \leq 12$, $k \geq 2$, and $\lambda\left(C_{9} \otimes C_{12 k}\right) \leq 12, k \geq 1$.

We have also found 12- $L(2,1)$-labelings of $C_{9} \otimes C_{49}, C_{9} \otimes$ $C_{50}, C_{9} \otimes C_{63}, C_{9} \otimes C_{29}, C_{9} \otimes C_{66}, C_{9} \otimes C_{55}, C_{9} \otimes C_{32}, C_{9} \otimes C_{33}$, $C_{9} \otimes C_{58}$, and $C_{9} \otimes C_{59}$. Any of these labelings restricted to first 12 copies of $C_{9}$ induces a 12-L(2,1)-labeling of $C_{9} \otimes C_{12}$. From Lemma 2 it follows that $\lambda\left(C_{9} \otimes C_{12 k+1}\right) \leq 12$ for $k \geq 4$, $\lambda\left(C_{9} \otimes C_{12 k+2}\right) \leq 12$ for $k \geq 4, \lambda\left(C_{9} \otimes C_{12 k+3}\right) \leq 12$ for $k \geq 5$, $\lambda\left(C_{9} \otimes C_{12 k+5}\right) \leq 12$ for $k \geq 2, \lambda\left(C_{9} \otimes C_{12 k+6}\right) \leq 12$ for $k \geq 5$, $\lambda\left(C_{9} \otimes C_{12 k+7}\right) \leq 12$ for $k \geq 4, \lambda\left(C_{9} \otimes C_{12 k+8}\right) \leq 12$ for $k \geq 2$, $\lambda\left(C_{9} \otimes C_{12 k+9}\right) \leq 12$ for $k \geq 2, \lambda\left(C_{9} \otimes C_{12 k+10}\right) \leq 12$ for $k \geq 4$, and $\lambda\left(C_{9} \otimes C_{12 k+11}\right) \leq 12$ for $k \geq 4$.

Since we have also found 12- $L(2,1)$-labelings of $C_{9} \otimes C_{30}$, $C_{9} \otimes C_{34}, C_{9} \otimes C_{37}, C_{9} \otimes C_{38}, C_{9} \otimes C_{39}, C_{9} \otimes C_{42}, C_{9} \otimes C_{43}, C_{9} \otimes C_{46}$, $C_{9} \otimes C_{47}, C_{9} \otimes C_{51}$, and $C_{9} \otimes C_{54}$, we establish that $\lambda\left(C_{9} \otimes C_{m}\right) \leq$ 12 for all $m$ of interest and the proof is complete. 
Theorem 18. If $m \geq 10$, then

$$
\lambda\left(C_{10} \otimes C_{m}\right)= \begin{cases}13, & m=10,11,13,15,16,17,22,26 \\ 12 \text { or } 13, & 27 \leq m \leq 395 \\ 12, & \text { otherwise. }\end{cases}
$$

Proof. We can see in Table 2 that $\lambda\left(C_{10} \otimes P_{5}\right)=12$ hence, it follows by Lemma 1 that $\lambda\left(C_{10} \otimes C_{m}\right) \geq 12$. The results for $m \leq 26$ are given in Table 2 .

The following pattern represents a $12-L(2,1)$-labeling of $C_{10} \otimes C_{37}$, while the leftmost 12 columns of the pattern represents a $12-L(2,1)$-labeling of $C_{10} \otimes C_{12}$ :

\begin{abstract}
$\begin{array}{lllllllllllllllllllllllllllllllllllll}8 & 3 & 1 & 12 & 8 & 5 & 7 & 2 & 0 & 11 & 9 & 6 & 8 & 3 & 1 & 12 & 10 & 7 & 4 & 2 & 0 & 11 & 9 & 6 & 8 & 3 & 1 & 12 & 10 & 5 & 7 & 4 & 2 & 0 & 11 & 9 & 6\end{array}$
$\begin{array}{llllllllllllllllllllllllllllllllllllll}0 & 11 & 9 & 6 & 3 & 1 & 12 & 10 & 5 & 7 & 4 & 2 & 0 & 11 & 9 & 6 & 3 & 1 & 12 & 10 & 5 & 7 & 4 & 2 & 0 & 11 & 9 & 6 & 8 & 3 & 1 & 12 & 10 & 5 & 7 & 4 & 2\end{array}$

$\begin{array}{lllllllllllllllllllllllllllllllllllll}7 & 4 & 2 & 0 & 11 & 9 & 6 & 8 & 3 & 1 & 12 & 10 & 5 & 7 & 2 & 0 & 11 & 9 & 6 & 8 & 3 & 1 & 12 & 10 & 5 & 7 & 4 & 2 & 0 & 11 & 9 & 6 & 8 & 3 & 1 & 12 & 10\end{array}$

$\begin{array}{llllllllllllllllllllllllllllllllllllll}1 & 12 & 10 & 5 & 7 & 4 & 2 & 0 & 11 & 9 & 6 & 3 & 1 & 12 & 10 & 5 & 7 & 4 & 2 & 0 & 11 & 9 & 6 & 8 & 3 & 1 & 12 & 10 & 5 & 7 & 4 & 2 & 0 & 11 & 9 & 6 & 3\end{array}$

$\begin{array}{llllllllllllllllllllllllllllllllllllll}9 & 6 & 8 & 3 & 1 & 12 & 10 & 5 & 7 & 2 & 0 & 11 & 9 & 6 & 8 & 3 & 1 & 12 & 10 & 5 & 7 & 4 & 2 & 0 & 11 & 9 & 6 & 8 & 3 & 1 & 12 & 10 & 5 & 7 & 2 & 0 & 11\end{array}$

$\begin{array}{llllllllllllllllllllllllllllllllllllll}4 & 2 & 0 & 11 & 9 & 6 & 3 & 1 & 12 & 10 & 5 & 7 & 4 & 2 & 0 & 11 & 9 & 6 & 8 & 3 & 1 & 12 & 10 & 5 & 7 & 4 & 2 & 0 & 11 & 9 & 6 & 3 & 1 & 12 & 10 & 5 & 7\end{array}$

$\begin{array}{llllllllllllllllllllllllllllllllllllll}12 & 10 & 5 & 7 & 4 & 0 & 11 & 9 & 4 & 8 & 3 & 1 & 12 & 10 & 5 & 7 & 4 & 2 & 0 & 11 & 9 & 6 & 8 & 3 & 1 & 12 & 10 & 5 & 7 & 2 & 0 & 11 & 9 & 6 & 8 & 3 & 1\end{array}$

$\begin{array}{llllllllllllllllllllllllllllllllllllll}6 & 8 & 3 & 1 & 12 & 8 & 5 & 7 & 2 & 0 & 11 & 9 & 6 & 8 & 3 & 1 & 12 & 10 & 5 & 7 & 4 & 2 & 0 & 11 & 6 & 8 & 3 & 1 & 12 & 10 & 5 & 7 & 4 & 2 & 0 & 11 & 9\end{array}$

$\begin{array}{llllllllllllllllllllllllllllllllllllll}2 & 0 & 11 & 9 & 6 & 3 & 1 & 12 & 10 & 5 & 7 & 4 & 2 & 0 & 11 & 9 & 6 & 8 & 3 & 1 & 12 & 10 & 7 & 4 & 2 & 0 & 11 & 9 & 6 & 8 & 3 & 1 & 12 & 10 & 5 & 7 & 4\end{array}$

$\begin{array}{llllllllllllllllllllllllllllllllllllll}10 & 5 & 7 & 4 & 0 & 11 & 9 & 4 & 8 & 3 & 1 & 12 & 10 & 5 & 7 & 4 & 2 & 0 & 11 & 6 & 8 & 3 & 1 & 12 & 10 & 5 & 7 & 4 & 2 & 0 & 11 & 9 & 6 & 8 & 3 & 1 & 12\end{array}$
\end{abstract}

By Lemma 2, we have $\lambda\left(C_{10} \otimes C_{12 \alpha+37 \beta}\right) \leq 12$ for integers $\alpha$ and $\beta$. Finally, thanks to Lemma 3 , we get $\lambda\left(C_{10} \otimes C_{m}\right) \leq 12$ for $m \geq(37-1) \cdot(12-1)=396$.

We have found 13- $L(2,1)$-labelings of $C_{10} \otimes C_{m}$ for $27 \leq$ $m \leq 46$ and we can construct $13-L(2,1)$-labelings of $C_{10} \otimes C_{m}$ for $m \geq 36$ as follows. We have found 13-L(2,1)-labelings of $C_{10} \otimes C_{25}, C_{10} \otimes C_{50}, C_{10} \otimes C_{27}, C_{10} \otimes C_{28}, C_{10} \otimes C_{29}, C_{10} \otimes$ $C_{54}$, and $C_{10} \otimes C_{39}$. Any of these labelings restricted to the first eight copies of $C_{10}$ is a 13- $L(2,1)$-labeling of $C_{10} \otimes C_{8}$. From Lemma 2 it follows that $\lambda\left(C_{10} \otimes C_{8 k}\right) \leq 13$ for $k \geq 1$, $\lambda\left(C_{10} \otimes C_{8 k+1}\right) \leq 13$ for $k \geq 3, \lambda\left(C_{10} \otimes C_{8 k+2}\right) \leq 13$ for $k \geq$ $6, \lambda\left(C_{10} \otimes C_{8 k+3}\right) \leq 13$ for $k \geq 3, \lambda\left(C_{10} \otimes C_{8 k+4}\right) \leq 13$ for $k \geq 3, \lambda\left(C_{10} \otimes C_{8 k+5}\right) \leq 13$ for $k \geq 3, \lambda\left(C_{10} \otimes C_{8 k+6}\right) \leq 13$ for $k \geq 6$, and $\lambda\left(C_{10} \otimes C_{8 k+7}\right) \leq 13$ for $k \geq 4$. These observations complete the proof.

Proposition 19. $\lambda\left(C_{11} \otimes C_{m}\right)=10$ only if $m \equiv 0(\bmod 11)$.

Proof. The graph $D_{11,10}^{2}$ with 380 vertices and the largest outdegree 2 has been computed. Since breadth first search algorithm has found only cycles of length length 11, Theorem 9 yields the proof.

Theorem 20. If $m \geq 11$, then

$$
\lambda\left(C_{11} \otimes C_{m}\right)= \begin{cases}10, & m \equiv 0(\bmod 11) \\ 12, & m \in\{12,13,14,15,16,18,19 \\ 11 & 20,21,22,23,25,26\} \\ 11 \text { or } 12, & \text { otherwise. }\end{cases}
$$

Proof. For $m \in\{12,13,14,15,16,18,19,20,21,22,23,25,26\}$ the $\lambda$ numbers are obtained by using the SAT reduction as depicted in Table 1 . Since $\lambda\left(C_{11} \otimes P_{3}\right)=10$, from Lemma 1 it follows that $\lambda\left(C_{11} \otimes C_{m}\right) \geq 10$, while from Proposition 19 it follows that $\lambda\left(C_{11} \otimes C_{m}\right) \geq 11$ if $m \neq \equiv(\bmod 11)$.
The result for $m \equiv 0(\bmod 11)$ can be obtained by the fact that $\lambda\left(C_{11} \otimes C_{11}\right)=10$ and by Lemma 2 .

Figure 6 represents an 11-L(2,1)-labeling of $C_{11} \otimes C_{28}$, where the leftmost 11 columns of the figure represent an 11-L(2,1)-labeling of $C_{11} \otimes C_{11}$. By Lemma 2 , we have $\lambda\left(C_{11} \otimes\right.$ $\left.C_{11 \alpha+28 \beta}\right) \leq 11$ for integers $\alpha$ and $\beta$. Finally, thanks to Lemma 3, we get $\lambda\left(C_{11} \otimes C_{m}\right) \leq 11$ for $m \geq(28-1) \cdot(11-1)=$ 270.

In order to find the general upper bound, we present the constructions showing that $\lambda\left(C_{11} \otimes C_{m}\right) \leq 12$ for $m \geq 26$. In particular, Figure 7 shows a $12-L(2,1)$-labeling of $C_{11} \otimes C_{37}$. This labeling restricted to the first 12 copies of $C_{11}$ induces a 12- $L(2,1)$-labeling of $C_{11} \otimes C_{12}$. From Lemma 2 it follows that $\lambda\left(C_{11} \otimes C_{12 k+1}\right) \leq 12, k \geq 3$, and $\lambda\left(C_{11} \otimes C_{12 k}\right) \leq 12, k \geq 1$.

Analogously, we have found 12- $L(2,1)$-labelings of $C_{11} \otimes$ $C_{38}, C_{11} \otimes C_{39}, C_{11} \otimes C_{40}, C_{11} \otimes C_{41}, C_{11} \otimes C_{42}, C_{11} \otimes C_{43}$, $C_{11} \otimes C_{44}, C_{11} \otimes C_{45}, C_{11} \otimes C_{46}$, and $C_{11} \otimes C_{35}$. Any of these labelings restricted to the first 12 copies of $C_{11}$ is a $12-L(2,1)$ labeling of $C_{11} \otimes C_{12}$.

From Lemma 2 it follows that $\lambda\left(C_{11} \otimes C_{12 k+2}\right) \leq 12$ for $k \geq 3, \lambda\left(C_{11} \otimes C_{12 k+3}\right) \leq 12$ for $k \geq 3, \lambda\left(C_{11} \otimes C_{12 k+4}\right) \leq 12$ for $k \geq 3, \lambda\left(C_{11} \otimes C_{12 k+5}\right) \leq 12$ for $k \geq 3, \lambda\left(C_{11} \otimes C_{12 k+6}\right) \leq 12$ for $k \geq 3, \lambda\left(C_{11} \otimes C_{12 k+7}\right) \leq 12$ for $k \geq 3, \lambda\left(C_{11} \otimes C_{12 k+8}\right) \leq 12$ for $k \geq 3, \lambda\left(C_{11} \otimes C_{12 k+9}\right) \leq 12$ for $k \geq 3, \lambda\left(C_{11} \otimes C_{12 k+10}\right) \leq 12$ for $k \geq 3$, and $\lambda\left(C_{11} \otimes C_{12 k+11}\right) \leq 12$ for $k \geq 2$.

Since we have also found 12- $L(2,1)$-labelings of $C_{11} \otimes C_{27}$, $C_{11} \otimes C_{28}, C_{11} \otimes C_{29}, C_{11} \otimes C_{30}, C_{11} \otimes C_{31}, C_{11} \otimes C_{32}, C_{11} \otimes C_{33}$, and $C_{11} \otimes C_{34}$, we establish that $\lambda\left(C_{11} \otimes C_{m}\right) \leq 12$ for all $m \geq 27$ and the proof is complete.

\section{3. $\lambda$-Numbers of $P_{n} \otimes C_{m}$}

Proposition 21. If $m \geq 3$, then

$$
\lambda\left(P_{3} \otimes C_{m}\right)= \begin{cases}14, & m=5 \\ 11, & m \in\{3,4,6,7,8,9,10,13,14,18,19\} \\ 10, & \text { otherwise. }\end{cases}
$$




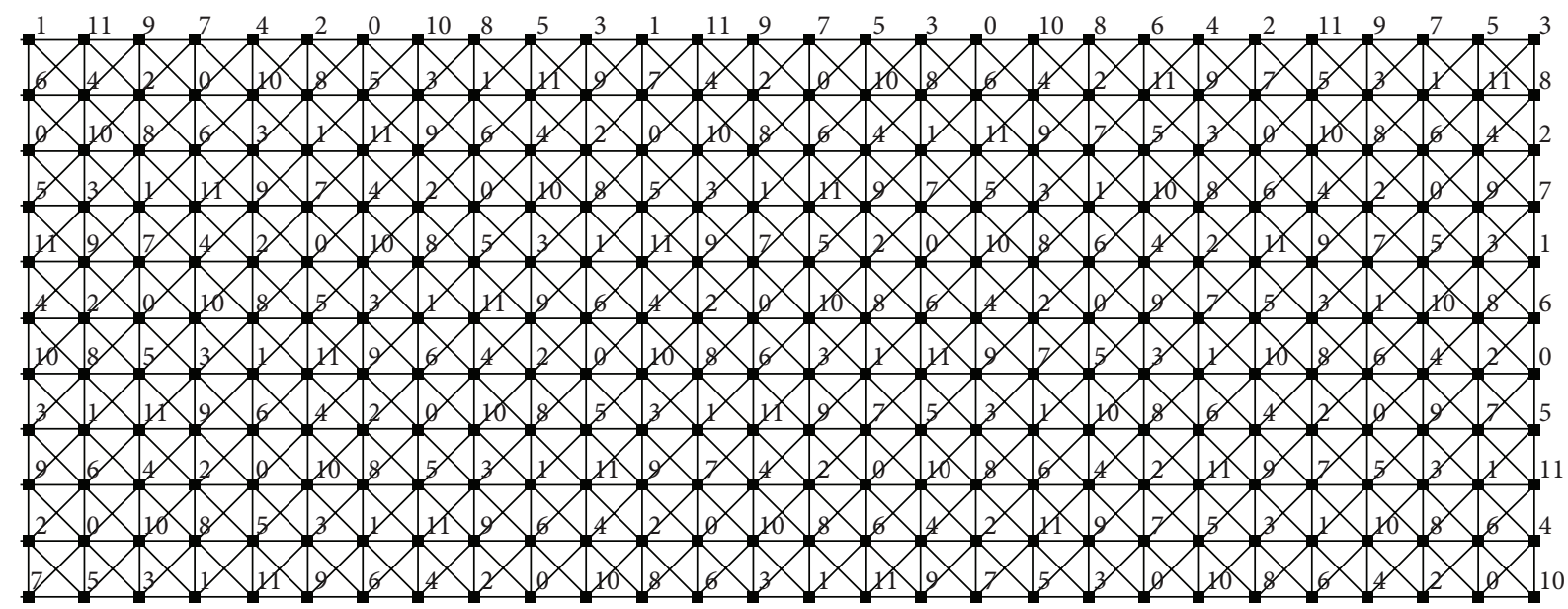

Figure 6: 11- $L(2,1)$-labeling of $C_{11} \otimes C_{28}$.

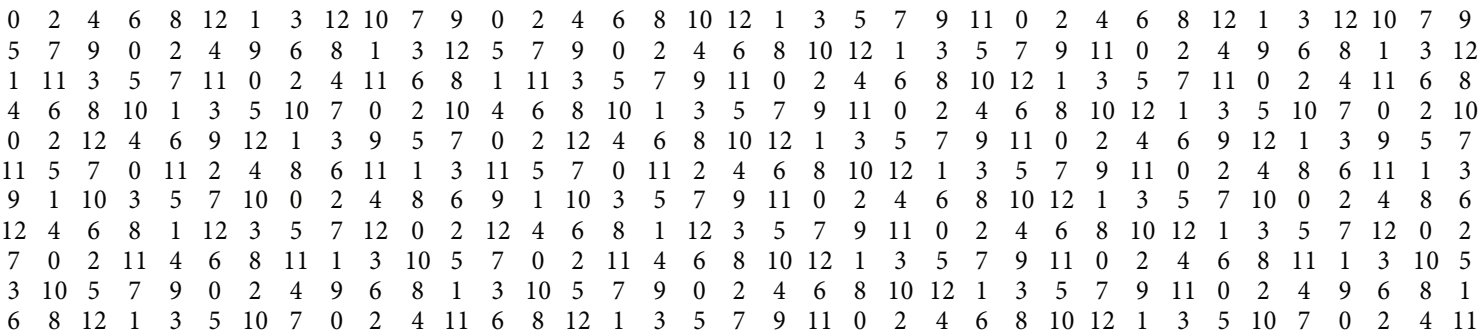

Figure 7: 12- $L(2,1)$-labeling of $C_{11} \otimes C_{37}$.

Proof. For $m \leq 12$ the results are obtained by solving the SAT instances transformed from the corresponding $L(2,1)$ labeling problems (see Table 1).

The graph $D_{3,10}^{\prime 2}$ with 9080 vertices and the largest outdegree 16 has been created in order to find $10-L(2,1)$-labelings in $P_{3} \otimes C_{m}$. Matrix multiplication has been applied in order to find closed directed walks in the graph. The algorithm has found no closed directed walk of length from the set $\{3,4,6,7,8,9,10,13,14,18,19\} \cup\{5\}$. It follows that $\lambda\left(P_{3} \otimes\right.$ $\left.C_{n}\right) \geq 11$ for any $m \in\{3,4,6,7,8,9,10,13,14,18,19\}$. The upper bounds for $m \in\{13,14,18,19\}$ follow from the labelings depicted in Figure 8.

We have found 10-L(2,1)-labelings of $P_{3} \otimes C_{23}, P_{3} \otimes C_{35}$, $P_{3} \otimes C_{47}, P_{3} \otimes C_{26}, P_{3} \otimes C_{38}, P_{3} \otimes C_{39}, P_{3} \otimes C_{40}, P_{3} \otimes C_{41}, P_{3} \otimes C_{31}$, and $P_{3} \otimes C_{43}$. Any of these labelings restricted to the first 11 copies of $P_{3}$ is a 10-L(2,1)-labeling of $P_{3} \otimes C_{11}$. From Lemma 2 it follows that $\lambda\left(P_{3} \otimes C_{11 k}\right) \leq 10$ for $k \geq 1, \lambda\left(P_{3} \otimes C_{11 k+1}\right) \leq 10$ for $k \geq 2, \lambda\left(P_{3} \otimes C_{11 k+2}\right) \leq 10$ for $k \geq 3, \lambda\left(P_{3} \otimes C_{11 k+3}\right) \leq 10$ for $k \geq 4, \lambda\left(P_{3} \otimes C_{11 k+4}\right) \leq 10$ for $k \geq 2, \lambda\left(P_{3} \otimes C_{11 k+5}\right) \leq 10$ for $k \geq 3, \lambda\left(P_{3} \otimes C_{11 k+6}\right) \leq 10$ for $k \geq 3, \lambda\left(P_{3} \otimes C_{11 k+7}\right) \leq 10$ for $k \geq 3, \lambda\left(P_{3} \otimes C_{11 k+8}\right) \leq 10$ for $k \geq 3, \lambda\left(P_{3} \otimes C_{11 k+9}\right) \leq 10$ for $k \geq 2$, and $\lambda\left(P_{3} \otimes C_{11 k+10}\right) \leq 10$ for $k \geq 3$.

Since we have also found 10-L(2,1)-labelings of $P_{3} \otimes C_{13}$, $P_{3} \otimes C_{14}, P_{3} \otimes C_{15}, P_{3} \otimes C_{16}, P_{3} \otimes C_{17}, P_{3} \otimes C_{18}, P_{3} \otimes C_{19}, P_{3} \otimes C_{20}$, $P_{3} \otimes C_{21}, P_{3} \otimes C_{24}, P_{3} \otimes C_{25}, P_{3} \otimes C_{27}, P_{3} \otimes C_{28}, P_{3} \otimes C_{29}, P_{3} \otimes C_{30}$,
$P_{3} \otimes C_{32}$, and $P_{3} \otimes C_{36}$, we establish that $\lambda\left(P_{3} \otimes C_{m}\right) \leq 10$ for all $m \geq 13$ and the proof is complete.

Proposition 22. If $m \geq 3$, then

$$
\lambda\left(P_{4} \otimes C_{m}\right)= \begin{cases}14, & m=5 \\ 10, & m \equiv 0(\bmod 11) \\ 11, & \text { otherwise. }\end{cases}
$$

Proof. For $m=5$ the result is obtained by solving the SAT instance transformed from the corresponding $L(2,1)$ labeling problem. For $m \equiv 0(\bmod 11)$ the result follows from Lemma 2 and from the fact that $\lambda\left(C_{11} \otimes C_{11}\right)=10$.

In order to find 10-L(2,1)-labelings in $P_{4} \otimes C_{m}$, the graph $D_{4,10}^{\prime 2}$ with 16792 vertices and the largest outdegree 3 has been created. Since breadth first search algorithm has found only cycles of length 11, the upper bound follows.

For all $m \neq \equiv 0(\bmod 11)$ and $m \geq 13$ we can construct 11-L(2,1)-labelings of $P_{4} \otimes C_{m}$ as described below. We have found 11-L(2, 1)-labelings of $P_{4} \otimes C_{13}, P_{4} \otimes C_{14}, P_{4} \otimes C_{15}, P_{4} \otimes$ $C_{16}$, and $P_{4} \otimes C_{17}$. Any of these labelings restricted to the first six copies of $P_{4}$ is an 11-L(2,1)-labeling of $P_{4} \otimes C_{11}$. From Lemma 2 it follows that $\lambda\left(P_{4} \otimes C_{6 k+1}\right) \leq 11$ for $k \geq 2, \lambda\left(P_{4} \otimes\right.$ $\left.C_{6 k+2}\right) \leq 11$ for $k \geq 2, \lambda\left(P_{4} \otimes C_{6 k+3}\right) \leq 11$ for $k \geq 2, \lambda\left(P_{4} \otimes\right.$ $\left.C_{6 k+4}\right) \leq 11$ for $k \geq 2$, and $\lambda\left(P_{4} \otimes C_{6 k+5}\right) \leq 11$ for $k \geq 2$. These conclusions complete the proof. 


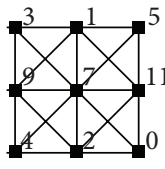

(a)

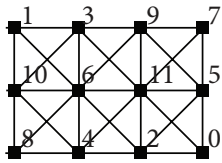

(b)

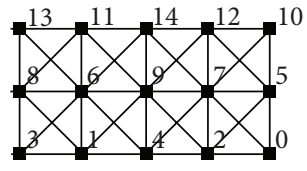

(c)

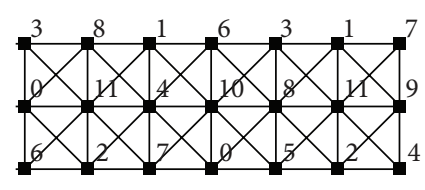

(d)

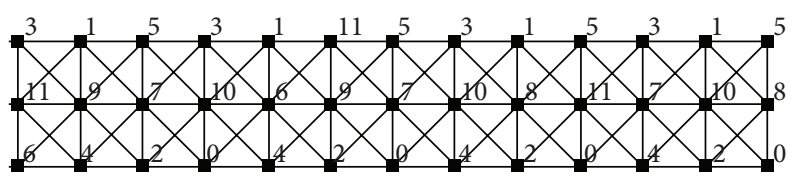

(e)

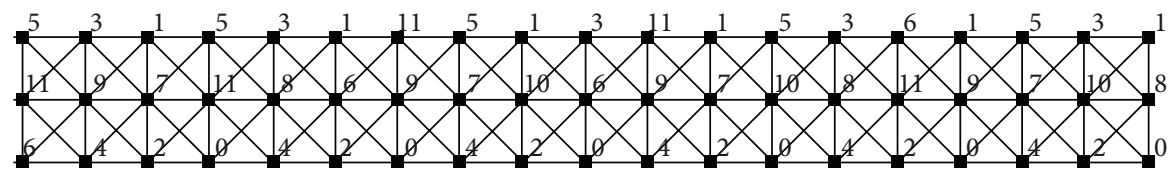

(f)

Figure 8: (a) 11- $L(2,1)$-labeling of $P_{3} \otimes C_{3}$, (b) 11- $L(2,1)$-labeling of $P_{3} \otimes C_{4}$, (c) 14- $L(2,1)$-labeling of $P_{3} \otimes C_{5}$, (d) 11-L(2,1)-labeling of $P_{3} \otimes C_{7}$, (e) 11- $L(2,1)$-labeling of $P_{3} \otimes C_{13}$, and (f) 11- $L(2,1)$-labeling of $P_{3} \otimes C_{19}$.

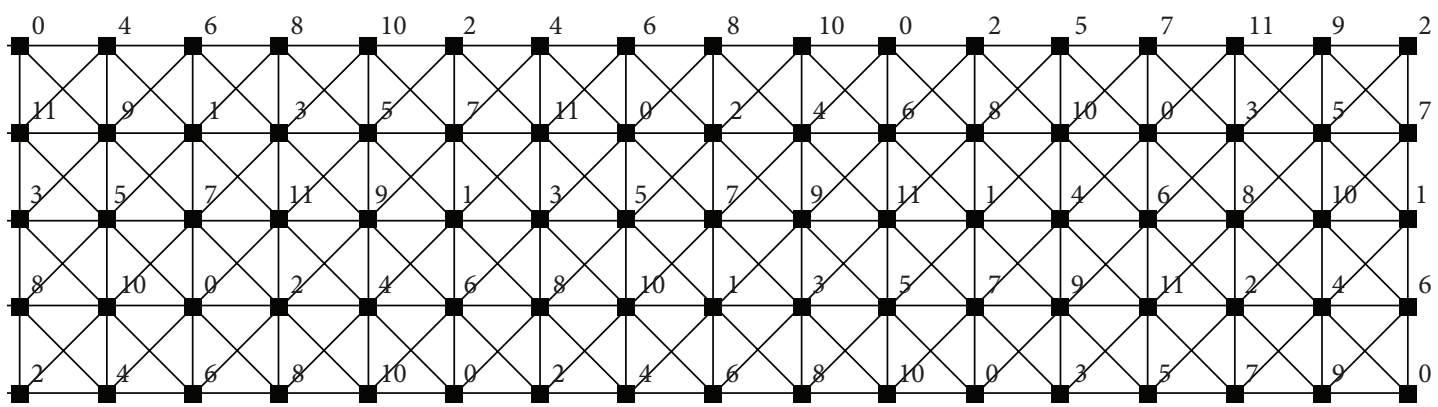

FIgURE 9: 11- $L(2,1)$-labeling of $P_{5} \otimes C_{17}$.

Corollary 23. Let $n, m \geq 4$.

(i) If $m \neq \equiv 0(\bmod 11)$, then $\lambda\left(P_{n} \otimes C_{m}\right) \geq 11$,

(ii) If $n \neq \equiv 0(\bmod 11)$ or $m \neq \equiv 0(\bmod 11)$, then $\lambda\left(C_{n} \otimes\right.$ $\left.C_{m}\right) \geq 11$.

From Theorem 6 now we have the following

Corollary 24. If $m \geq 24$ and $n \geq 26$, then

$$
\lambda\left(C_{n} \otimes C_{m}\right)= \begin{cases}10, & n \equiv 0(\bmod 11) \\ 11 \text { or } 12, & \text { otherwise. }\end{cases}
$$

Proposition 25. If $m \geq 3$, then

$$
\lambda\left(P_{5} \otimes C_{m}\right)= \begin{cases}14, & m=5 \\ 12, & m=3,9,10 \\ 10, & m \equiv 0(\bmod 11) \\ 11, & m=4,6,7,8,12 \text { or } m \geq 80 \\ 11 \text { or } 12, & \text { otherwise. }\end{cases}
$$

Proof. For $m \leq 12$ the results follow from Table 1 . For $m \equiv$ $0(\bmod 11)$ the result follows from Lemma 2 and from the fact that $\lambda\left(C_{11} \otimes C_{11}\right)=10$.

Figure 9 represents an $11-L(2,1)$-labeling of $P_{5} \otimes C_{17}$, where the leftmost six columns of the figure represent an 11- $L(2,1)$-labeling of $P_{5} \otimes C_{6}$.

By Lemma 2, we have $\lambda\left(P_{5} \otimes C_{6 \alpha+17 \beta}\right) \leq 11$ for integers $\alpha$ and $\beta$. Finally, thanks to Lemma 3 , we get $\lambda\left(P_{5} \otimes C_{m}\right) \leq 11$ for $m \geq(17-1) \cdot(6-1)=80$.

In order to complete the proof note that from Theorem 16 and Lemma 1 it follows that $\lambda\left(P_{5} \otimes C_{m}\right) \leq 12$ for $m \geq 11$.

Proposition 26. If $m \geq 3$, then

$$
\lambda\left(P_{6} \otimes C_{m}\right)= \begin{cases}14, & m=5 \\ 12, & m=3,9,10 \\ 10, & m \equiv 0(\bmod 11) \\ 11, & m=4,6,7,8,12 \text { or } m \geq 154 \\ 11 \text { or } 12, & \text { otherwise. }\end{cases}
$$




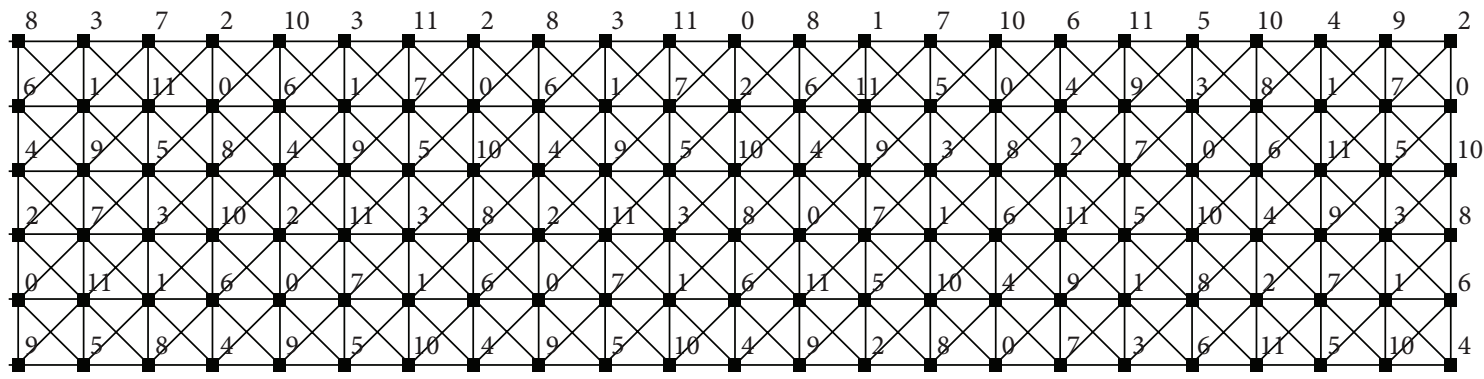

Figure 10: 11- $L(2,1)$-labeling of $P_{6} \otimes C_{23}$.

Proof. For $m \leq 12$ the results follow from Table 1. For $m \equiv$ 0 (mod11) the result follows Lemma 2 and from the fact that $\lambda\left(C_{11} \otimes C_{11}\right)=10$.

Figure 10 represents a $11-L(2,1)$-labeling of $P_{6} \otimes C_{23}$, where the leftmost eight columns of the figure represent an 11-L(2,1)-labeling of $P_{6} \otimes C_{8}$.

By Lemma 2, we have $\lambda\left(P_{6} \otimes C_{8 \alpha+23 \beta}\right) \leq 11$ for integers $\alpha$ and $\beta$. From Lemma 3 it follows that $\lambda\left(P_{6} \otimes C_{m}\right) \leq 11$ for $m \geq(23-1) \cdot(8-1)=154$.

We complete the proof by noting that from Theorem 16 and Lemma 1 it follows $\lambda\left(P_{6} \otimes C_{m}\right) \leq 12$ for $m \geq 11$.

Values in Table 1, the results from Section 4.2, Theorems 6 and 20, and Corollary 23 provide lower and upper bounds for the $\lambda$-number of $P_{n} \otimes C_{m}$. The results are summarized in the following.

Theorem 27. If $n \geq 7$, then

$$
\lambda\left(P_{n} \otimes C_{m}\right)= \begin{cases}10, & m \equiv 0(\bmod 11) \\ 14, & m=5 \\ 12, & m=3,7,9,10 \\ 11, & m=4,6,8,12 \text { or } m \geq 270 \\ 11 \text { or } 12, & \text { otherwise. }\end{cases}
$$

\section{Conclusion}

In this paper, the $L(2,1)$-labeling problem of the strong product of paths and cycles is studied. The problem derives from the more general Frequency Assignment Problem (FAP) which requires assigning frequencies to transmitters in a wireless network. It is well known that some interesting wireless networks are closely connected to the strong product of graphs. For example, an octagonal grid is the strong product of two paths and an octagonal torus is the strong product of two cycles.

By using various computational approaches, we succeed in solving the problem (except for the final number of cases) for the strong product of a path and a cycle, as well as for the the strong product of two cycles, where one of the cycles is of length at most eleven. Moreover, the obtained results enable us to improve the bounds on the $\lambda$-number for the strong product of two cycles, where both cycles are sufficiently long.
Finding the exact $\lambda$-numbers for these graphs is therefore an interesting and challenging avenue of further research.

\section{Conflict of Interests}

The authors wish to confirm that there is no known conflicts of interests associated with this paper and there has been no significant financial support for this work that could have influenced its outcome. They confirm that the paper has been read and approved by all named authors and that there are no other persons who satisfied the criteria for authorship but are not listed. They further confirm that the order of authors listed in the paper has been approved by all of us. They confirm that they have given due consideration to the protection of intellectual property associated with this work and that there are no impediments to publication, including the timing of publication, with respect to intellectual property. In so doing they confirm that they have followed the regulations of their institutions concerning intellectual property.

\section{Acknowledgments}

Aleksander Vesel is supported by the Ministry of Science of Slovenia under the Grant 0101-P-297. Zehui Shao is supported by the National Natural Science Foundation of China under the Grant 61309015.

\section{References}

[1] J. Kratochvíl, D. Kratsch, and M. Liedloff, "Exact algorithms for $L(2,1)$-labeling of graphs," in Proceedings of the 32nd Mathematical Foundations of Computer Science (MFCS '07), pp. 513-524, 2007.

[2] L. M. San José-Revuelta, "A new adaptive genetic algorithm for fixed channel assignment," Information Sciences, vol. 177, no. 13, pp. 2655-2678, 2007.

[3] G. J. Chang and D. Kuo, "The $L(2,1)$-labeling problem on graphs," SIAM Journal on Discrete Mathematics, vol. 9, no. 2, pp. 309-316, 1996.

[4] J. R. Griggs and R. K. Yeh, "Labelling graphs with a condition at distance two," SIAM Journal on Discrete Mathematics, vol. 5, pp. 586-595, 1992.

[5] H. L. Bodlaender, T. Kloks, R. B. Tan, and J. Van Leeuwen, "Approximations for $\lambda$-colorings of graphs," Computer Journal, vol. 47, no. 2, pp. 193-204, 2004. 
[6] J. Fiala, T. Kloks, and J. Kratochvíl, "Fixed-parameter complexity of $\lambda$-labelings," Discrete Applied Mathematics, vol. 113, pp. 59-72, 2001.

[7] J. Fiala, P. A. Golovach, and J. Kratochvíl, "Distance-constrained labelings of graphs of bounded treewidth," in Proceedings of the 32nd International Colloquium on Automata, Languages and Programming (ICALP '05), pp. 360-372, 2005.

[8] R. Hammack, W. Imrich, and S. Klavžar, Handbook of Product Graphs, CRC Press, Boca Raton, Fla, USA, 2nd edition, 2011.

[9] N. Kumar, M. Kumar, and R. B. Patel, "Capacity and interference aware link scheduling with channel assignment in wireless mesh networks," Journal of Network and Computer Applications, vol. 34, no. 1, pp. 30-38, 2011.

[10] S. Klavžar and A. Vesel, "Computing graph invariants on rotagraphs using dynamic algorithm approach: the case of $(2,1)$ colorings and independence numbers," Discrete Applied Mathematics, vol. 129, pp. 449-460, 2003.

[11] M. A. Whittlesey, J. P. Georges, and D. W. Mauro, "On the $\lambda$ number of $Q_{n}$ and related graphs," SIAM Journal on Discrete Mathematics, vol. 8, pp. 499-506, 1995.

[12] C. Schwarz and D. S. Troxell, " $L(2,1)$-labelings of Cartesian products of two cycles," Discrete Applied Mathematics, vol. 154, no. 10, pp. 1522-1540, 2006.

[13] P. K. Jha, S. Klavžar, and A. Vesel, " $L(2,1)$-labeling of direct product of paths and cycles," Discrete Applied Mathematics, vol. 145, pp. 317-325, 2005.

[14] P. K. Jha, "Optimal $L(2,1)$-labeling of strong products of cycles," IEEE Transactions on Circuits and Systems I, vol. 48, no. 4, pp. 498-500, 2001.

[15] D. Korže and A. Vesel, " $L(2,1)$-labeling of strong products of cycles," Information Processing Letters, vol. 94, no. 4, pp. 183190, 2005.

[16] J. J. Sylvester, "Mathematical questions with their solutions," Educational Times, vol. 41, pp. 171-178, 1884.

[17] Z. Shao and A. Vesel, "Integer linear programming model and SAT reduction for distance-constrained labelings of graphs: the case of $L(3,2,1)$-labeling for products of paths and cycles," IET Communications, vol. 7, no. 8, pp. 715-720, 2013.

[18] D. A. Wheeler, "MiniSAT User Guide: How to use the MiniSAT SAT Solver," http://www.dwheeler.com/essays/minisat-userguide.html. 

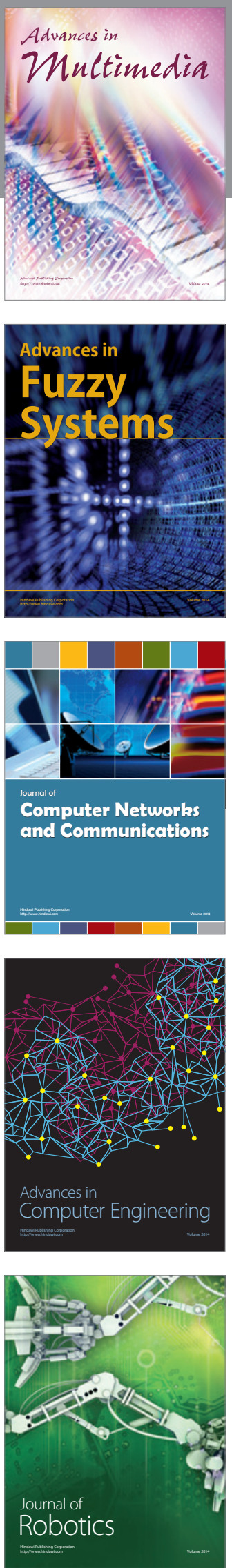

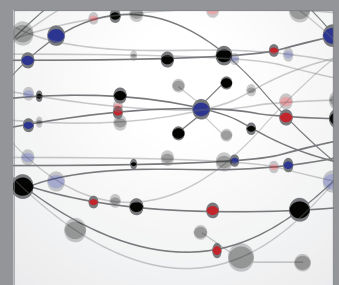

The Scientific World Journal
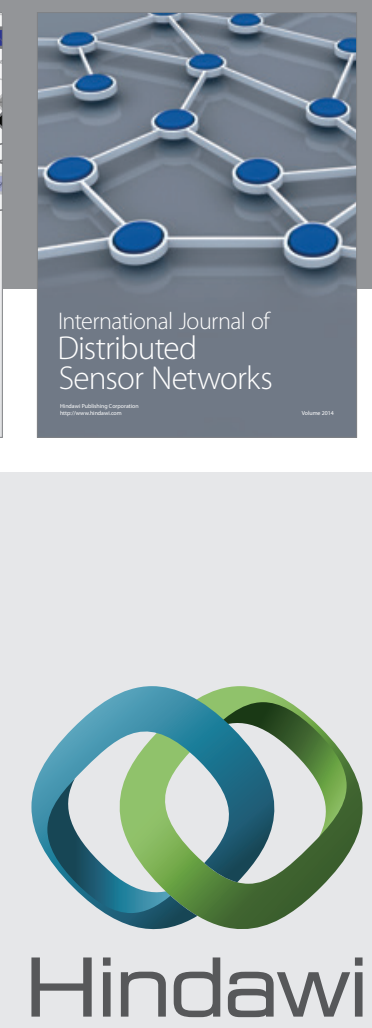

Submit your manuscripts at

http://www.hindawi.com
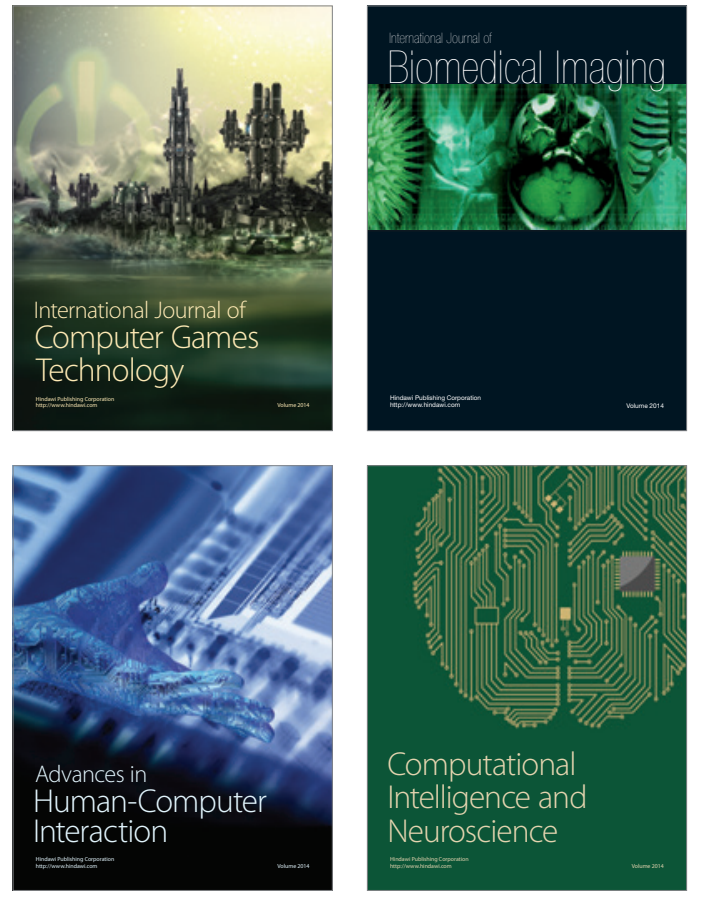
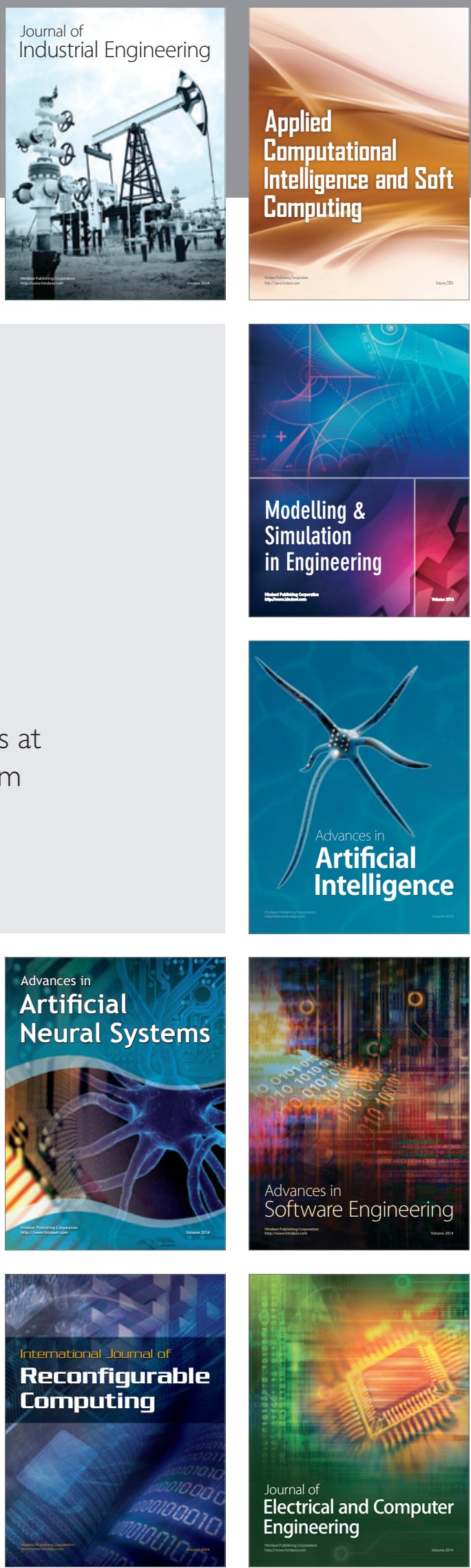